\title{
REPRESENTACIONES SOBRE SEXISMO EN EDUCACIÓN SUPERIOR Y FORMACIÓN PROFESIONAL
}

\author{
(REPRESENTATIONS OF SEXISM IN HIGHER EDUCATION AND PROFESSIONAL \\ TRAINING)
}

Patricia Fernández Rotaeche

Joana Jaureguizar Alboniga-Mayor

Nahia Idoiaga Mondragon

Universidad del País Vasco (UPV/EHU)

DOI: $10.5944 / e d u c X X 1.28225$

\begin{abstract}
Cómo referenciar este artículo/How to reference this article:
Fernández Rotaeche, P., Jaureguizar Alboniga-Mayor, J., e Idoiaga Mondragon, N., (2021). Representaciones sobre sexismo en Educación Superior y Formación Profesional. Educación XX1, 24(2), 421-440. https://doi.org/10.5944/educXX1.28225
\end{abstract}

Fernández Rotaeche, P., Jaureguizar Alboniga-Mayor, J., \& Idoiaga Mondragon, N., (2021). Representations of sexism in Higher Education and Professional Training. Educación XX1, 24(2), 421-440. https://doi.org/10.5944/educXX1.28225

\section{RESUMEN}

La educación, y la escuela como institución, juegan un papel fundamental en el proceso de socialización de todo ser humano, y, a través de ella se transmiten creencias y actitudes donde se constata el papel del personal docente como transmisor de las mismas. Entre las creencias y actitudes que el profesorado puede transmitir de forma no consciente están las ideas sexistas. Este estudio quiere conocer la representación que sobre el sexismo tiene el alumnado de grados de Educación Infantil, Primaria y Técnico Superior en Educación Infantil, considerando que estos son el futuro personal docente-educador y, por tanto, responsables de la transmisión de ideas, creencias y actitudes en las aulas. Para llevar a cabo esta investigación, 114 alumnas y alumnos de grados de Educación Infantil y Primaria, así como también alumnado de formación profesional, Técnicos y Técnicas en Educación Infantil, realizaron una tarea de 
asociación libre mediante el Grid Elaboration Method con el fin de recoger las representaciones sobre sexismo de dicho alumnado. Para analizar el contenido del ejercicio se realizó un análisis lexical mediante el método Reinert a través del software Iramuteq. Los resultados más significativos nos muestran que este alumnado representa el sexismo como una infravaloración por razón de género, y que esta infravaloración se daría por igual en hombres y en mujeres. El estudio también recoge situaciones sexistas que el alumnado observa y vive, las cuales son identificadas en su mayoría en el ámbito laboral y deportivo, quedando excluido el sexismo en el ámbito educativo. Finalmente, se quiere conocer las repercusiones sociales del sexismo, donde sobre todo se menciona la discriminación social hacia la mujer, haciendo claras referencias al patriarcado. Además, se discute la ausencia de conciencia de este alumnado de que el sexismo se puede dar también en el ámbito educativo, y sobre las implicaciones de la misma.

\section{PALABRAS CLAVE}

Educación, sexismo, profesorado, género

\section{ABSTRACT}

Education and school play a fundamental role in the process of socialization of every human being, and, through it, beliefs and attitudes are transmitted where the role of the teaching staff as a transmitter of the same is verified. Among the beliefs and attitudes that teachers can transmit unconsciously are sexist ideas. This study wants to understand the representation among the students of Infant Education degree, Primary Education degree and Top Technician of Infant Education, because they are the future teacher-educator staff and, therefore, responsible for the transmission of ideas, beliefs and attitudes in the classroom. To undertake this investigation, 114 students from Infant Education, Primary Education and Higher Technician of Infant Education performed a free association task through the Grid Elaboration Method in order to collect the representations on sexism. To analyse the content of the exercise, a lexical analysis was performed using the Reinert method through the Iramuteq software. The most significant results show that these students represent sexism as a gender-based undervaluation, and that underestimation would occur equally in men and women. The study also includes sexist situations that students observe and experience, which are identified in the work and sports field, surprisingly excluding the educational field. Finally, we want to know the social repercussions of sexism. In this research, social discrimination against women is mentioned with clear references to the patriarchy. In addition, the lack of awareness of these students that sexism can also occur in the educational field and its implications are discussed. 


\section{KEYWORDS}

Education, sexism, teacher, gender

\section{INTRODUCCIÓN}

En nuestra sociedad, aunque en materia de igualdad de género sean muchas cosas las que hayan cambiado y los modelos de género hayan ido transformándose con los años, la discriminación, la injusticia y la dominación cobran nuevas y sutiles formas (Ballarín, 2017). Si bien durante las últimas décadas estamos asistiendo a numerosos avances en materia de igualdad entre mujeres y hombres, está constatada la permanencia del sexismo en nuestra sociedad (Herrero et al., 2017) y por ende también en nuestras instituciones educativas, ya que estas forman parte de la sociedad, siendo también un reflejo de ellas. Este estudio se lleva a cabo con alumnado de grados de Educación Infantil y Primaria, así como con alumnado de Formación Profesional, Técnicos y Técnicas en Educación infantil, con el objetivo de explorar, por un lado, cómo representan el sexismo, y, por otro lado, las experiencias sexistas vividas y observadas. Este alumnado es el futuro inmediato de la educación, el futuro profesorado, y, por tanto, en las aulas además de transmitir contenido académico, transmitirán también su ideología y sus valores sociales y culturales (Giddens, 2001). Estudios recientes nos revelan que la igualdad de género está prácticamente ausente en grados de Educación Infantil y Primaria (Ballarín, 2017; Gónzález-Pérez, 2017; Gudbjornsdottir, et al., 2017; Kreitz-Sandberg, 2013; Lahelma y Tainio, 2019; Valdivieso, 2016), y que, además, para el alumnado de estos grados, el género es un tema secundario (González-Pérez, 2017; Vizcarra, et al., 2015). Dada esta situación, no nos debe extrañar que el sexismo tenga cabida, aun hoy día, en nuestras instituciones educativas.

Por todo ello, consideramos necesario dejar evidencias de las representaciones sobre sexismo que tiene este alumnado invitando a que, desde dichas evidencias, futuras investigaciones pudieran diseñar trabajos de intervención con el de fin de transformar las representaciones sobre sexismo que tiene nuestro alumnado. Por otra parte, no debemos olvidar que el contexto educativo también puede ser un lugar donde se aprende y desarrolla la capacidad crítica y el pensamiento, y que el profesorado también tiene un papel activo como agente idóneo para cuestionar la desigualdad de género y para construir nuevos modelos de lo que es ser un hombre o ser una mujer.

Tradicionalmente, el sexismo ha sido considerado una actitud de prejuicio hacia las mujeres y una de las principales actitudes que mantiene las desigualdades entre sexos (Moya, 2004). Además, este hace referencia a un conjunto de creencias que aparecen como resultado de la desigual representación social de hombres y mujeres, y esta representación se articula conformando toda una ideología de género donde ni la sexualidad, ni la familia, ni las relaciones humanas, vienen de 
la naturaleza, sino de la cultura social, donde en algún momento de la Historia las mujeres se sometieron a los hombres (Vidal, 2019). Actualmente, la agenda política de la ideología de género se propone cambiar esta situación histórica, deconstruyendo (reconstruyendo) todo lo humano, es decir, suprimiendo cualquier distinción entre lo femenino y lo masculino, entre otras muchas cuestiones.

En la actualidad, el sexismo se define como una actitud dirigida hacia las personas en virtud de su pertenencia a un determinado sexo biológico donde se asumen diferentes características y conductas (Lameiras, 2002). Del sexismo se desprende la idea de que hombres y mujeres son diferentes y, por tanto, deben adherirse a un conjunto de normas y comportamientos sociales específicos de acuerdo al género que se les presupone. Estas creencias instauran la superioridad de un género (el masculino) frente a otro (el femenino), perpetuando un sistema donde se mantienen las desigualdades de género (Doob, 2015) y los comportamientos discriminatorios hacia las mujeres (Cuadrado, 2009). Desde esa línea, investigadoras como Díaz Aguado (2006) señalan que el sexismo está constituido por tres componentes. Por un lado, estaría el componente cognitivo, formado por el conjunto de erróneas creencias donde las diferencias de sexo son la causa de todas las diferencias sociales y psicológicas; por otro lado, estaría el componente afectivo-valorativo, donde se asocian los valores de fuerza, poder, y violencia a lo masculino, y la sumisión y debilidad a lo femenino; y, por último, estaría el componente conductual, el cual incluye la tendencia a llevar a la práctica las creencias y los valores a través de conductas violentas en el caso de los hombres y de sumisión en el caso de las mujeres.

Recientes estudios, como los de Aguaded (2017) o Esteban y Fernández (2017), han puesto de manifiesto que el sexismo persiste en la juventud española, la cual es una etapa de la vida en la que hay una reestructuración social y una consolidación de la identidad. Por ello, se convierte en una etapa vulnerable para el desarrollo de actitudes y creencias sexistas. Diversas investigaciones coinciden en estas cuestiones, aunque no está claro cómo evolucionan estas creencias (Díaz et al. 2010; Moya y Expósito, 2001). Las características más relevantes encontradas en la etapa juvenil relacionadas con la actitud sexista son la dificultad para reconocer las desigualdades de género, tendiendo a ser atribuidas a diferencias biológicas (Díaz-Aguado, 2003). Además, estudios como los de León y Aizpurúa (2020), Esteban y Fernández Montaño (2017) y Garaigordobil (2015), confirman que hay diferencias de género en el sexismo, donde los hombres son significativamente más sexistas que las mujeres y se evidencia también que las mujeres continúan siendo las que son valoradas en base a estereotipos sexistas de género en mayor medida que los hombres.

\section{Transmisión del sexismo en la escuela}

El centro educativo puede entenderse como entorno social con unas reglas, códigos, valores, etc., en relación con el género. Investigadores como 
Guerrero et al. (2006), resuelven que la escuela cumple un importante papel en la socialización de género, constituyéndose como un agente que continúa reproduciendo estereotipos y pautas sociales tradicionales. García-Pérez et al. (2010) revelan también que la organización escolar está, entre otras cuestiones, determinada por las creencias sociales sobre género. Podemos decir que, hoy en día, en las escuelas se defiende el currículo coeducativo, aunque hay autores que afirman que la cultura sexista aún domina en las aulas (Araya Umaña, 2003). En los centros educativos, es evidente que el profesorado tiene un papel relevante en la transmisión de dicha cultura (Rebollo et al., 2011), en la que las ideas, las creencias y las actitudes pueden ser sexistas, por tanto, se hace necesario comprender la posición y sensibilidad que muestran con respecto a la igualdad de género para poder ejecutar proyectos de acción educativa (McCormack y Gleeson, 2010).

Autores como Colás y Jiménez (2006) identifican incluso niveles de conciencia del profesorado sobre género que afectan a la capacidad para percibir y reconocer prácticas y situaciones de desigualdad y discriminación. Por tanto, parece necesario trabajar en la detección de creencias y actitudes sexistas que pudiera tener el futuro personal docente-educador con el fin de poder transformarlas a través de sendas intervenciones educativas con contenidos relativos a la igualdad de género, los cuales redundarían en una disminución de los niveles de sexismo (Cárdenas et al., 2010; Castro et al., 2010; Lemus et al., 2008; Torres et al., 2005), además de demostrarse que las jóvenes y los jóvenes que cursan estudios vinculados a la mujer poseen menores niveles de sexismo y estereotipos de género (Jenaro et al., 2014).

Teniendo en cuenta todo lo anterior, resulta importante analizar las representaciones o creencias que tienen los jóvenes de hoy en día en cuanto al sexismo. De ese modo, nos acercaremos a comprender cómo estas se pueden transmitir en el futuro, tanto en las aulas como en otros ámbitos de interacción social. Teniendo en cuenta que entre la juventud existe una escasa conciencia sobre los riesgos y el comportamiento sexista (Díaz Aguado, 2013; Silva, et al., 2017), se podría pensar que esta juventud también tendrá una escasa conciencia sobre el riesgo que supone la transmisión de ideas sexistas a las nuevas generaciones desde el ámbito educativo y social.

A pesar de la importancia de la igualdad de género en todos los ámbitos de interacción social, y en especial en el ámbito educativo, este no ha emergido como una prioridad en los planes de actuación de la educación superior (Anguita Martínez, 2011; Lahelma y Tainio, 2019; Zippel, et al., 2016). Así, investigaciones como la de Garrigues (Grupo de Investigación Isonomia, 2010), y Larrondo y Rivero (2019), reflejan bien la resistencia a la inclusión del género en el currículum universitario. Lombardo y Mergaert (2013) concluyen que hay una resistencia que puede tomar forma institucional o individual, y que esta niega la necesidad de cambio, y resta importancia a las políticas de igualdad. 
Así, el objetivo general del presente estudio es explorar las representaciones sobre el sexismo del alumnado de grados de Educación Infantil y Primaria, así como de alumnado de Formación Profesional, Técnicos y Técnicas en Educación infantil. Los objetivos específicos del estudio son los siguientes:

- Describir, mediante un ejercicio de libre asociación, qué es lo que el alumnado en formación para ser futuro docente piensa sobre el sexismo.

- Recoger las experiencias sexistas observadas y vividas por las personas participantes.

- Explorar si las personas jóvenes participantes manifiestan ser conscientes del riesgo de la transmisión de ideas y actitudes sexistas en el ámbito educativo.

\section{METODOLOGÍA}

\section{Participantes}

La muestra estuvo formada por 114 estudiantes ( 88 mujeres y 26 hombres). Los criterios de inclusión fueron: ser mayor de edad (18 o más años) y estar estudiando algún grado universitario o de Formación Profesional que habilite para ser docente/educador de Educación Infantil o Primaria. 92 participantes fueron de los de los Grados de Educación Primaria y Educación Infantil de las Facultades de Educación de Álava y Vizcaya de la Universidad del País Vasco (UPV/EHU), y, 22 participantes del $1{ }^{\circ}$ curso de Técnico Superior en Educación Infantil de un Instituto de Educación Secundaria (IES) de Vizcaya. La media de edad fue de $19.73, D T=3.50$, con un rango de edad que oscilaba entre 18 y 28 años.

\section{Procedimiento}

Los datos de este estudio fueron recogidos los meses de noviembre y diciembre de 2019. Tras haber obtenido el permiso de las instituciones correspondientes y del profesorado y alumnado implicado, se procedió a la administración del cuestionario. El alumnado cumplimentó el cuestionario en presencia de una de las investigadoras, la cual estaba presente en el aula para cualquier aclaración sobre el mismo. La participación fue voluntaria, anónima y confidencial y representaba al $80.17 \%$ del alumnado de grados universitarios de Educación Infantil y Primaria; y el $19.3 \%$ de $1^{\circ}$ curso de Técnico Superior en Educación Infantil. Finalmente, fueron entregados y cumplimentados un total de 114 cuestionarios. 
Este estudio fue aprobado por el Comité de Ética en la Investigación con Seres Humanos (CEISH) de la Universidad del País Vasco (UPV/EHU).

\section{Diseño}

El presente estudio sigue un diseño cualitativo basándose en la asociación libre de palabras que se considera un método idóneo para reunir los pensamientos y sentimientos naturalistas que la gente tiene sobre temas específicos. Además, se lleva a cabo el análisis del corpus mediante el método Reinert. Este método se basa en la premisa de que las palabras no son independientes unas de otras, sino que reflejan una forma de pensar sobre el objeto del que se habla, ya que es a partir de ellas que los enunciados adquieren significado (Reinert, 1996).

\section{Instrumento}

Para analizar las representaciones sobre sexismo se utilizó el Grid Elaboration Method libre asociación (Joffe y Elsey, 2014). El objetivo de este método es conseguir una explicación detallada sobre la representación del sexismo de cada participante. Siguiendo este método, se les proporcionó a todas las personas participantes un documento con instrucciones y otra hoja con cuatro casillas en blanco. En las instrucciones se les pidió que escribieran las cuatro primeras palabras o ideas que les vinieran a la mente al oir la palabra "sexismo". Se pidió a todas las personas que rellenasen las cuatro casillas. Posteriormente, se les pidió que explicasen por escrito cada una de las palabras o ideas que habían mencionado lo más profundamente posible (por lo menos 2-3 párrafos por palabra). De esta manera se obtuvo un corpus de palabras con una descripción completa y estructurada de las cuatro representaciones más importantes ligadas al sexismo de cada participante.

\section{Análisis de datos}

El corpus de respuestas se analizó mediante el Método Reinert de análisis lexical con el software Iramuteq (Reinert, 1996). Específicamente, el software divide el corpus inicial en Unidades Contextuales Elementales o ECUs, los cuales tienen una longitud aproximada de 30 a 50 palabras, y dicho corpus se analiza considerando qué palabras completas hay en cada ECU. Estas palabras son utilizadas para crear una tabla de contingencia que muestra la distribución del vocabulario por ECU. Partiendo de esta tabla de contingencia, el programa genera una matriz cuadrada de distancias, lo que implica que dos ECUs estarán cerca entre sí, si comparten algunas de las palabras analizadas (Reinert, 1996). A continuación, se realiza un análisis jerárquico descendente para clasificar y 
agrupar las ECUS en clases y conocer el vocabulario más característico de cada una de ellas.

Mediante este proceso, el software ayuda a la interpretación de textos, ya que extrae clases o grupos de palabras que ocurren simultáneamente y están diferenciadas del resto. Los criterios para la inclusión de cada forma, siguieron los indicados por los estudios previos (Camargo y Bousfield, 2009), y fueron: 1) frecuencia superior al promedio de ocurrencias en el corpus; 2) un valor esperado de la palabra superior a 3; 3) asociación con la clase determinada por el valor de chi-cuadrado igual o superior a 3.89, Esto proporciona un margen de error $\mathrm{p}<0.05(\mathrm{df}=1)$.

Así, se obtienen una serie de clases formadas por palabras y segmentos de texto (ECUs) típicos con valores estadísticos significativos. Esto proporciona la base estadística para interpretar las clases o los principales temas tratados en el corpus. Una vez identificadas las clases, se asocian con variables "pasivas" (variables independientes), en el presente caso, el género de los participantes (mujer, hombre u otros) y el tipo de estudios que estaban cursando (universitario vs formación profesional). Las operaciones en el método Reinert son estadísticas, transparentes y reproducibles hasta el momento de la interpretación, en el que las investigadoras asignan un nombre a cada una de las clases basándose en el vocabulario específico y segmentos típicos de la misma propuestos por el software, apoyándose en coocurrencias y patrones de distribución (SchonhardtBailey, 2013).

\section{RESULTADOS}

\section{Representación sobre el sexismo}

El corpus completo estuvo formado por 7393 palabras y 1190 de ellas eran palabras únicas. El análisis jerárquico descendente segmentó el texto en 215 ECUs.

Como se puede observar en el dendograma de la Figura 1, siguiendo la división del análisis de clúster, se extrajeron seis clases o temáticas principales referidas a cómo representa el sexismo el alumnado. 
Figura 1

Dendograma de la Clasificación Jerárquica Descendente de la representación del sexismo

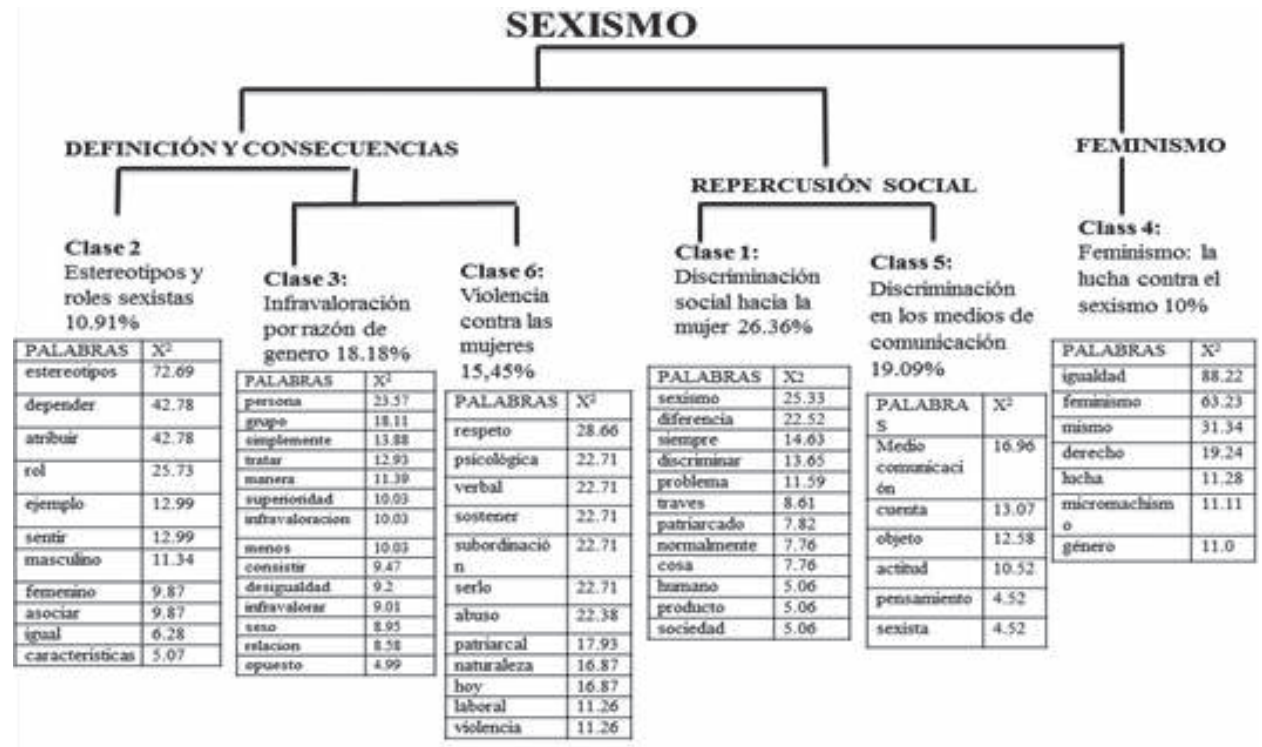

El dendograma se dividió en tres ramas: la primera rama se compone de la segunda, tercera y sexta clase, las cuales primeramente definían el sexismo y después las consecuencias de este. Concretamente, en la clase 3 se define el sexismo como la "Infravaloración por razón de género" (18.18\%). En esta representación del sexismo, curiosamente el alumnado recalca la discriminación por género pero no la discriminación de la mujer explícitamente: "El sexismo es apartar o menospreciar a una persona por sus pensamientos, ideas o simplemente por las ideas que se tienen cerca de un grupo de personas antes de conocerlas" (mujer, universitaria; $X^{2}=93.75$ ); "el sexismo es una actitud de superioridad hacia una persona por una característica diferente" (mujer, universitaria; $X^{2}=$ 92.08); "el sexismo es la desigualdad en el trato hacia los hombres y las mujeres, la sociedad trata de manera distinta al individuo si este es hombre o mujer" (hombre, universitario; $X^{2}=72.31$ ).

En la segunda clase, siguiendo con el patrón anterior, el alumnado define los "Estereotipos y roles sexistas" (10.91\%). Como se puede observar en los segmentos más característicos, se hace especial hincapié en los estereotipos masculinos: "El azul para el chico al igual que los coches, y dependiendo del sexo que tenga la persona se le asocia un tipo de comportamiento, asociar el género a un sexo determinado sin importar cómo se sienta la persona" (hombre, universitario; $X^{2}=141.27$ ); "Por ejemplo, si un chico no sabe arreglar un coche, característica asignada al sexo masculino, va a ser infravalorado por la sociedad, el sexismo determina diferentes características a cada sexo y por ello se forman 
estereotipos acerca de lo que se debe hacer; depende de si eres hombre o mujer" (mujer, universitaria; $X^{2}=179.39$ ).

Para acabar con esta rama, emerge la sexta clase que liga el sexismo a la violencia contra las mujeres (15.45\%). En esta clase, la referencia a las mujeres como víctimas del sexismo son claras: "La agresión tanto psicológica como física que hoy en día las mujeres reciben por el simple hecho de serlo (mujer, universitaria; $X^{2}=184.28$ ); “Los abusos sexuales están cada vez más presentes, pero, incluso hoy en día, cuesta que se reconozcan” (hombre, universitario; $X^{2}=$ 102.37); “ La discriminación de género por parte del hombre hacia la mujer, y el hacer daño intencionado físico o psicológico”( hombre, universitario, $X^{2}=79.66$ ).

En una segunda rama del dendograma, tenemos a la primera y la quinta clase donde el alumnado reflexionó sobre la repercusión social que puede tener el sexismo. En la primera clase encontramos los siguientes ejemplos: "Discriminación social hacia la mujer" (26.36\%) en la que el alumnado va más allá de la definición genérica que daba en la tercera clase del sexismo y remarca que el sexismo es una problemática social, real y aplicada que desemboca en la discriminación hacia las mujeres por el mero hecho de serlo. En algún ejemplo encontramos una clara referencia al patriarcado como sistema social y político donde sitúan al hombre en el centro-antropocentrismo: "El sexismo discrimina a la mujer en la sociedad y el sexismo suele estar vinculado al predominio de los hombres en la sociedad" (mujer, universitaria; $X^{2}=67.37$ ).

Esta primera clase está unida en el dendograma a la quinta clase, denominada "Discriminación en los medios de comunicación" (19.09\%), reflejo de cómo el sexismo se hace especialmente visible y fuerte en la sociedad a través de los medios: "Prácticamente todos los medios de comunicación ponen a la mujer como un objeto o un trofeo haciendo parecer inferior al hombre" (mujer, formación profesional; $\left.X^{2}=96.14\right)$; "Gran parte de la sociedad tiene inmerso el sexismo en sus cabezas, esto se debe a los mensajes que se transmiten en los medios de comunicación, los valores de las familias tradicionales y la explotación de la mujer la mujer como objeto" (mujer, universitaria; $X^{2}=92.32$ ). Aun así, algunos segmentos también mencionan la importancia de los medios para visibilizar y dar a conocer la discriminación que viven las mujeres: "En los medios de comunicación se habla de diferencias salariales entre hombres y mujeres desempeñando el mismo trabajo" (mujer, universitaria; $X^{2}=86.95$ ).

Por último, la tercera rama se denominó "Feminismo: la lucha contra el sexismo" (10\%), donde aparece el feminismo como herramienta para hacer frente a la discriminación que puede crear el sexismo, como se menciona anteriormente: "La igualdad es que todas las personas de nuestra sociedad tengan los mismos derechos y que sean tratados con igualdad" (mujer, universitaria, $X^{2}=227.70$ ); "El feminismo es una lucha que se da por la igualdad de derechos entre hombres y mujeres"(mujer, universitaria, $X^{2}=195.89$ ). No obstante, también parece que se liga el feminismo a otro tipo de conceptos como el hembrismo: "El hembrismo 
nos lleva a que las mujeres tengan más derechos que los hombres, y el machismo a que los hombres tengan más derechos que las mujeres" (mujer, universitaria, $\left.X^{2}=225.34\right)$. Por último, cabe destacar que esta clase se halló relacionada significativamente a las mujeres $(\mathrm{p}<.005)$ y esta fue la única diferencia por sexo o tipo de estudios que se encontró.

\section{Situaciones sexistas identificadas}

El corpus completo estuvo formado por 9034 palabras y 1645 de ellas eran palabras únicas. El análisis jerárquico descendente segmentó el texto en 241 ECUs.

Como se puede observar en el dendograma de la Figura 2, siguiendo la división del análisis de clúster, se extrajeron cuatro clases o temáticas principales referidas a las situaciones o contextos sexistas identificadas por el alumnado.

Figura 2

Dendograma de la Clasificación Jerárquica Descendente de las situaciones o contextos sexistas identificados

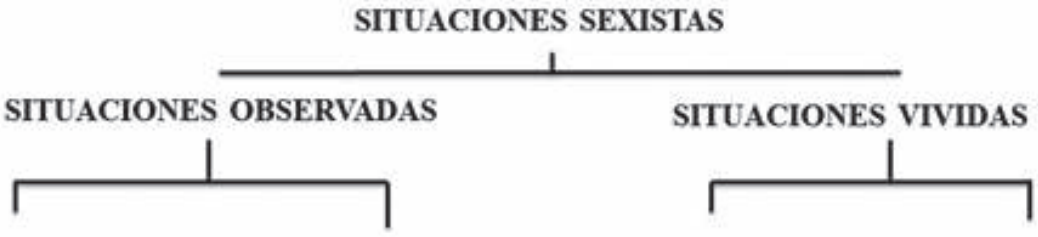

\begin{tabular}{|c|c|}
\hline PALABRAS & $\mathrm{X}^{2}$ \\
\hline menos & 16.38 \\
\hline cobrex & 15.05 \\
\hline $\min d 0$ & 10.05 \\
\hline boenbre & 10.05 \\
\hline matre & 8.24 \\
\hline trabojis & 8.2 \\
\hline casa & 798 \\
\hline bibcral & 798 \\
\hline conducir & 7,92 \\
\hline ewherazar & 7.34 \\
\hline mrjer & 696 \\
\hline trabojer & 6.78 \\
\hline Contratar & 6.54 \\
\hline Inportancia & 5.93 \\
\hline puesto & 593 \\
\hline
\end{tabular}

Clase 4: Sexismo en los medios de comunicación $17.42 \%$

\begin{tabular}{|c|c|}
\hline PAL.ABRAS & $\mathrm{X}^{2}$ \\
\hline anncia & 29,07 \\
\hline televisiön & 18.98 \\
\hline jugacte & 14.55 \\
\hline colego & 14.55 \\
\hline ropa & 14.55 \\
\hline pecueito & 14,14 \\
\hline prodacto & 14.14 \\
\hline femetaine & 12.03 \\
\hline comentario & 9.76 \\
\hline masculino & 9.5 \\
\hline déferencia & 9.5 \\
\hline sexo & 8.1 \\
\hline Bebero & 8.1 \\
\hline sociodad & 6.67 \\
\hline
\end{tabular}

Clase 2:

Sexismo en el deporte profesional y escolar $26.52 \%$

\begin{tabular}{|l|l|}
\hline PALABRAS & $\mathrm{X}^{2}$ \\
\hline jugar & 15.93 \\
\hline partido & 14.4 \\
\hline fitbol & 13.3 \\
\hline conpabero & 13.15 \\
\hline profesor & 13.03 \\
\hline rechazar & 11.43 \\
\hline chico & 10.41 \\
\hline amiso & 8.51 \\
\hline clase & 8.51 \\
\hline entresader & 8.51 \\
\hline ingtitar & 8.51 \\
\hline
\end{tabular}

Clase 3: Sexismo en la calle $21.97 \%$

\begin{tabular}{|l|l|}
\hline PALABRAS & $\mathrm{X}^{2}$ \\
\hline ectrar & 38.43 \\
\hline discoteca & 23.24 \\
\hline calle & 15.38 \\
\hline tecar & 14.65 \\
\hline fiesta & 13.17 \\
\hline consentimierto & 12.15 \\
\hline coche & 10.09 \\
\hline noche & 10.09 \\
\hline pagar & 10.61 \\
\hline piropo & 10.21 \\
\hline sufrir & 10.21 \\
\hline Gratis & 8.16 \\
\hline Descriminat & 7.33 \\
\hline Sibidos & 6.77 \\
\hline pucblo & 6.77 \\
\hline escuchar & 6.77 \\
\hline ertrada & 4.38 \\
\hline puta & 3.86 \\
\hline
\end{tabular}

El dendograma se dividió en dos ramas. En la primera rama emergen la primera y la cuarta clase, y en ellas el alumnado describe situaciones sexistas que 
ha observado en la sociedad, sobre todo ligadas al mundo laboral. La primera clase, hace referencia al "Sexismo vivido en el entorno laboral" (34.09\%), ligando el sexismo a la diferenciación salarial, al techo de cristal, a la discriminación a la mujer en el trabajo, etc., como se puede observar en los segmentos más significativos de esta clase: "Aun teniendo los mismo estudios y el mismo, cargo hay mujeres que cobran menos que los hombres, y además, a las mujeres se les cierran más las puertas del mercado laboral por el hecho de querer ser madres" (mujer, universitaria, $X^{2}=74.35$ ); "A las mujeres no las hacen fijas por el hecho de ser mujer" (mujer, universitaria, $X^{2}=73.72$ ). Además, algunos segmentos también hacen referencia a que todavía las tareas domésticas se consideran labor de las mujeres, perpetuando así el estereotipo de género: "Muchos hombres no quieren que sus mujeres trabajen porque en casa están mejor, planchando y cocinando para ellos" (mujer, universitaria, $X^{2}=74.35$ ).

Siguiendo con el sexismo observado, la cuarta clase hace referencia al "Sexismo en los medios de comunicación" (17.42\%). Se recogen los ejemplos que se consideran más relevantes como indicadores del sexismo latente en los medios, a los cuales el alumnado hace responsable de mantener los estereotipos y roles sexistas donde aparece una imagen degradada de la mujer: "En la televisión se puede ver publicidad sexista donde se asocia a la mujer con el cuidado familiar y la limpieza, y al hombre con los roles que se le asignan socialmente" (mujer, universitaria, $X^{2}=120.91$ ); "En los anuncios publicitarios a la hora de presentar productos para la limpieza siempre aparecen mujeres" (hombre, universitario, $\left.X^{2}=87.74\right)$; "La mujer en los medios de comunicación aparece como objeto, como producto, cosificada" (mujer, universitaria, $X^{2}=77.21$ ).

Por otro lado, en la identificación de situaciones sexistas hay una segunda rama donde el alumnado identifica situaciones que ha vivido mayoritariamente en primera persona. Para empezar, en la segunda clase nos hablan del sexismo en el deporte profesional y en el ámbito escolar (26.52\%). Los ejemplos que se mencionan son los siguientes: "El sexismo en el deporte se ve cuando se cree que a la mujer le va a costar mayor esfuerzo lograr los mismos resultados deportivos que a los hombres" (mujer, universitaria, $X^{2}=68.80$ ); "A las mujeres no se nos toma en cuenta para al fútbol por ejemplo" (mujer, universitaria, $\left.X^{2}=57.23\right)$; "En la escuela, cuando los niños jugábamos al fútbol y las niñas no tenían más remedio que quedarse hablando o cambiando pegatinas" (hombre, universitario, $X^{2}=40.39$ ).

Por último, la tercera clase se refiere al "Sexismo en la calle" (21.97\%) donde las mujeres cuentan cuáles son las situaciones sexistas que han vivido: "Cuando monto en el coche con mis amigas y amigos, estos dan por hecho que tienen que ser copilotos, y además las chicas muchas veces entran gratis a las discotecas y los chicos deben pagar la entrada" (hombre, universitario, $X^{2}=216.91$ ); "El sexismo en la calle se detecta en el momento que te piropean en la calle" (mujer, universitaria, $X^{2}=107.60$ ). Los segmentos típicos se recrudecen cuando las mujeres hablan sobre las situaciones sexistas que han sufrido en 
ambiente festivo: "El sexismo se vive cuando un chico te toca sin tu permiso, o por la noche, cuando fui por la calle y un chico me gritó: corre que te voy a pillar" (mujer, universitaria, $X^{2}=80.80$ ). Sin embargo, en esta clase algunos hombres han querido remarcar que ellos también viven situaciones sexistas de fiesta: "Las discotecas, cuando por ser chico y el aforo estaba casi lleno no me han dejado entrar, o me ha salido más caro, cuando muchas mujeres entraban gratis" (hombre, universitario, $X^{2}=107.6$ ).

\section{DISCUSIÓN Y CONCLUSIONES}

Este estudio ha tratado de proporcionar información específica acerca de lo que piensa sobre el sexismo el futuro profesorado de Educación infantil, Primaria y Formación Profesional.

El primer objetivo del presente estudio fue describir qué es lo que el alumnado, en formación para ser futuro docente, piensa sobre el sexismo. Los resultados más significativos nos revelan que el alumnado representa el sexismo como la infravaloración que se hace por razón de género, aunque la infravaloración que perciben no es exclusivamente hacia la mujer, sino indistintamente hacia el hombre o hacia la mujer; cuando se constata la clara desventaja social de la mujer con respecto al hombre por el simple hecho de ser mujer. Es decir, estos resultados no concuerdan con la literatura académica revisada en materia de género, que nos revela que, históricamente, las mujeres han sido estigmatizadas de manera negativa, ya sea porque se les ha achacado cierta inferioridad intelectual (Bosch et al., 1992), cierta debilidad moral (Sánchez, 1997), o porque se les relega a roles tradicionales, y que son comunicados como si se tratase de una realidad perpetua (Velandia-Morales y Rincón, 2014). Son resultados, cuando menos llamativos, y más aún si se tiene en cuenta que la muestra estaba compuesta mayoritariamente por mujeres. La referencia clara a las mujeres aparece cuando las personas participantes mencionan la violencia contra las mujeres. Así, y siguiendo la clasificación de Díaz Aguado (2006), se haría referencia al componente comportamental del sexismo, pero no al cognitivo. En cuanto al componente afectivo-valorativo del sexismo, se podría concluir que los/as participantes hacen referencia al mismo cuando aluden a los estereotipos sexistas.

El segundo objetivo del estudio fue recoger las experiencias sexistas observadas y vividas por el alumnado participante. Entre las experiencias manifestadas, destacan las situaciones de discriminación hacia la mujer en el entorno laboral. Estos resultados vendrían a corroborar la fotografía sobre la brecha salarial actual entre hombres y mujeres que muestra el Instituto Nacional de Estadística (INE, 2018), donde presenta una brecha salarial de género según ganancia media anual del $22.3 \%$ y según ganancia por hora normal de trabajo, del $14.7 \%$. Los datos mencionados evidencian una clara discriminación de la mujer en el ámbito laboral, y, desde esta línea, cabe mencionar los estudios 
recientes de Ferrant y Kolev (2016) que demuestran que la discriminación de las mujeres en general en las instituciones sociales dificulta el crecimiento económico en el largo plazo, y estiman que el efecto sobre el PIB global actual de la discriminación puede llegar ser del 16\%. Por lo tanto, esta desigualdad sería una cuestión a tener en cuenta si un país quiere crecer y desarrollarse en al ámbito económico.

El presente estudio destaca también la mención de sexismo en los medios de comunicación, en los cuales se trata a la mujer como objeto o como trofeo para los hombres, es decir, se evidencia el fenómeno de cosificación sexual del cuerpo de la mujer (Bartky, 1990 en Calogero et al., 2010; Fredrickson y Roberts, 1997). Estos resultados son coincidentes con los resultados del estudio de Mateos de Cabo, Gimeno Nógues y Martínez Martínez (2009) donde se estudian 34.235 noticias recogidas de la prensa española, y donde se concluye que los diarios digitales estudiados no solo siguen reproduciendo los estereotipos observados en la sociedad, sino que también pueden considerarse parte activa a la hora de construirlos. Se encontró que la proporción de mujeres citadas en la prensa digital es de un 18\% del total de personas mencionadas; la probabilidad de que la mujer aparezca mencionada se reduce drásticamente (alrededor del 7\%) cuando la noticia aparece en la sección de deportes. Esta cuestión de la discriminación en el ámbito deportivo también se refleja en nuestro estudio, donde el $26.52 \%$ menciona que se puede dar el trato desigual de la mujer con respecto al hombre tanto en el deporte escolar como en el profesional.

Finamente, cabe destacar una cuestión que nos parece relevante; en ningún momento se menciona que el sexismo se pueda dar en el ámbito educativo. Estos resultados coinciden con estudios previos ya citados, en los que se destaca la baja conciencia de estudiantes universitarios del ámbito educativo sobre las desigualdades de género en relación a los procesos instructivos (MirallesCardona et al., 2020). Este resultado iría unido al tercer y último objetivo del presente estudio, que trataba de explorar si las personas jóvenes participantes manifestaban ser conscientes del riesgo de la transmisión de ideas y actitudes sexistas en el ámbito educativo, siendo la respuesta claramente negativa. Esta falta de mención del ámbito educativo, nos llevaría a la siguiente reflexión: ¿cómo va a poder el profesorado detectar las ideas, las creencias y actitudes sexistas que pudieran transmitir a su alumnado si no hay una conciencia de que el sexismo se puede dar en el ámbito educativo y que el profesorado es un agente activo en la transmisión de ideas? Desde esta línea, Giddens (2001) y Pérez (2014) concluyen que la escuela, como agente de socialización, es una institución donde se puede transmitir sexismo, y que además, como señala también Moreno (2000), el profesorado puede transmitir ideas y actitudes sexistas con sus prácticas y con el currículum oculto. Esos procesos de transmisión pueden implementar diferencias respecto al género, como señalan Walker y Barton (1983), y Pérez (2014), y con esas diferencias fomentar las actitudes sexistas. Por lo tanto, llama la atención que las personas participantes de este estudio no hayan realizado esta reflexión, lo que nos podría llevar a una segunda observación: ¿se está formando 
adecuadamente al futuro profesorado de educación primaria, educación infantil y técnicos superiores en educación infantil para que sean agentes activos capaces de formar en igualdad y con actitud crítica ante el sexismo de nuestra sociedad? A este respecto, el estudio de Cordón et al. (2019) concluye que la formación inicial de los maestros y maestras no elimina adecuadamente las actitudes sexistas. Todo ello, por lo tanto, pondría en evidencia la necesidad de replantear la formación del profesorado en lo que a igualdad de género se refiere.

Concluimos diciendo que es fundamental continuar indagando y ofreciendo nuevas perspectivas y enfoques de análisis del sexismo que nos aporten una visión de la realidad para poder dotar tanto al profesorado en activo como al futuro profesorado de herramientas para la educar en igualdad (coeducación), para que la escuela sea una institución donde se aprenda y desarrolle la capacidad crítica y para que el profesorado tenga una clara conciencia de lo que transmite y de cómo lo transmite (López-Vélez, 2014). Por tanto, no debemos olvidar que para tener una escuela con un profesorado consciente de su influencia en el alumnado debemos de seguir trabajando en esta línea.

Para terminar, habría que señalar las limitaciones del presente estudio. La primera limitación haría referencia a la muestra, concretamente a su tamaño y a su procedencia: si bien se ha recogido muestra en diferentes instituciones de formación universitaria y formación profesional del País Vasco, obtener un mayor tamaño de muestra y ampliar la recogida a otras comunidades autónomas de España, contribuiría a su representatividad y a la generalización de los resultados. Por otro lado, sería interesante completar la recogida de información con otros instrumentos, tales como cuestionarios validados, e incluso contrastar los resultados con la visión de profesorado en activo, para tener una visión más completa y poder detectar posibles lagunas que se pudiesen cubrir en la formación de los futuros docentes. 


\section{REFERENCIAS BIBLIOGRÁFICAS}

Aguaded, E. M. (2017). Análisis de la presencia de sexismo en alumnado universitario. ENSAYOS, Revista de la Facultad de Educación de Albacete, 32(1), 127-143.

Anguita-Martínez, R. (2011). El reto de la formación del profesorado para la igualdad. Revista Interuniversitaria de Formación del Profesorado, 14(1), 43- 51.

Araya, S. (2003). Relaciones sexistas en la educación. Revista Educación, 27(1). 41-52.

Ballarín Domingo, P. (2017). ¿Se enseña coeducación en la Universidad? Atlánticas. Revista Internacional de Estudios Feministas, 2(1), 7-31. http:// dx.doi.org/10.17979/arief.2017.2.1.1865

Bosch, E. \& Ferrer, V. (2012). Nuevo mapa de los mitos sobre la violencia de género en el siglo XXI. Psicothema, 24(4), 548554.

Bosch, E., Manassero, M. A., \& Ferrer, V. A. (1992). La misoginia medieval y su repercusión en el concepto de enfermedad mental en la mujer. Revista de Historia de la Psicología, 13(2-3), 329334.

Calogero, R. M., Tantleff-Dunn, S., \& Thompson, J. K. (Eds.). (2011). Selfobjectification in women: Causes, consequences, and counteractions. American Psychological Association.

Camargo, B., \& Bousfield, A. B. (2009). Social representation, risk behaviors and AIDS. The Spanish Journal of Psychology, 12(2), 565-575.

Cárdenas, M., Lay, S. L., González, C., Calderón, C., \& Alegría, I. (2010). Inventario de sexismo ambivalente: Adaptación, validación y relación con variables psicosociales. Salud y Sociedad, 1(2), 125-135.

Castro, Y. R., Fernández, M. L., Fernández, M. V. C., \& Garrido, J. M. F. (2010).
Evaluación de las actitudes sexistas en estudiantes españoles/as de educación secundaria obligatoria. Psicología. Avances de la Disciplina, 4(1), 11-24.

Colás, P., \& Jiménez, R. (2006). Tipos de conciencia de género del profesorado en los contextos escolares. Revista de Educación, 340, 415-444.

Cordón, S., Gutiérrez-Esteban, P., \& Cubo, S. (2019). Development of sexist attitudes in primary school teachers during their initial teacher training. Women's Studies International Forum, 72, 32-39.

Cruz Torres, C. E., Zempoaltecalt Alonso. V., \& Correa Romero, F.E. (2005). Perfiles de sexismo en la ciudad de México: Validación del cuestionario de medición del sexismo ambivalente. Enseñanza e Investigación en Psicología, 10(2), 381-395.

Cuadrado, I. (2009). El estudio psicosocial del prejuicio. En E. Gaviria, I. Cuadrado, \& M. López-Sáez (Eds.), Introducción a la Psicología Social (pp. 387-423). Sanz y Torres.

Diaz, C., Rosas. M A., \& González, M. T. (2010). Escala de machismo sexual (EMS-Sexismo-12). Diseño y análisis de propiedades psicométricas, Summa Psicológica UST, 7(2), 35-44.

Díaz-Aguado, M. J. (Ed.) (2013). Evolución de la adolescencia española sobre la igualdad y prevención de la violencia de género. Ministerio de Sanidad, Servicios Sociales e Igualdad.

Díaz-Aguado, M. J. (2006). Sexismo, violencia de género y acoso escolar. Propuestas para una prevención integral de la violencia. Revista de Estudios de Juventud, 73, 38-57.

Díaz-Aguado, M. J. (2003). Adolescencia, sexismo y violencia de género. Papeles del Psicólogo, 23(84), 35-44. 
Díaz-Aguado, M.J., y Martínez- Arias, R. (2001). La construcción de la igualdad y la prevención de la violencia contra la mujer desde la educación secundaria. Ministerio de Trabajo y de Asuntos Sociales. Instituto de la Mujer.

Doob, C. B. (2015). Social inequality and social stratification in US society? Routledge. https://doi. org/10.4324/9781315662800

Esteban, B., \& Fernández, P. (2017). ¿Actitudes sexistas en jóvenes?: Exploración del sexismo ambivalente y neosexismo en población universitaria. Femeris, 2(2), 137-153. https://doi. org/10.20318/femeris.2017.3762

Ferrant, G., \& A. Kolev (2016). Does gender discrimination in social institutions matter for long-term growth? Cross-country evidence, $O E C D$ Development Centre Working Papers, 330. OECD Publishing. https://doi. org/10.1787/5jm2hz8dgls6-en.

Fredrickson, B., \& Roberts, T. (1997). Objectification Theory: Toward understanding women 凶s lived experiences and mental health risks. Psychology of Women Quarterly, 21(2), 173-206. https://doi. org/10.1111/j.1471-6402.1997.tb00108.x

Garaigordobil, M. (2015). Sexismo y expresión de la ira: Diferencias de género, cambios con la edad y correlaciones entre ambos constructos. Revista Argentina de Clínica Psicológica, 24(1), 35-42.

Grupo de Investigación Isonomia (2010). Proyecto integración de la perspectiva de género y las enseñanzas en materia de igualdad de mujeres y hombres y no discriminación en los planes de estudio de grado de la Universitat Jaume I. https:// n9.cl/n53m8

Guerrero, E., Valdés, A., \& Provoste, P. (2006). La desigualdad olvidada: Género y educación en Chile. En P. Provoste (Ed.),
Equidad de género y reformas educativas: Argentina, Chile, Colombia y Perú (pp. 99150). Hexagrama Consultores.

Giddens, A. (2001). Sociología. Editorial Alianza.

González-Pérez, T. (2017). Políticas educativas igualitarias en España: la igualdad de género en los estudios de magisterio. Archivos Analíticos de Políticas Educativas, 26(2), 1-17. https:// doi.org/10.14507/epaa.26.2764

Gudbjornsdottir, G.S., Thordardottir, T., \& Larusdottir, S.H. (2017). Gender equality issues in teacher education and in schools: A plea for a change in practice. [Comunicación de Congreso] Conferencia Gender Training in Education, Lisboa, Portugal.

Herrero, J., Rodríguez, F.J., \& Torres, A. (2017). Acceptability of partner violence in 51 societies: The role of sexism and attitudes toward violence in social relationships. Violence Against Women, 23(3), 351-367. https://doi. org/10.1177/1077801216642870

Jenaro, C., Flores, N., \& Castaño, R. (2014). Actitudes hacia la diversidad: el papel del género y de la formación. Cuestiones de género: de la igualdad y la diferencia, 9, $50-62$.

Joffe, H., \& Elsey, J.W. (2014). Free association in psychology and the grid elaboration method. Review of General Psychology, 18(3), 173-185.

Kreitz-Sandberg, S. (2013). Gender inclusion and horizontal gender segregation: Stakeholders' strategies and dilemmas in Swedish teachers' education. Gender and Education, 25(4), 444-465.

Lahelma, E., \& Tainio, L. (2019). The long mission towards gender equality in teacher education: Reflections from a national project in Finland. Nordic Studies in Education, 39(1), 69-84. 
Lameiras, M. (2002). El sexismo y sus dos caras: de la hostilidad a la ambivalencia. Anuario de Sexología, 8, 91-102.

Larrondo, A., \& Rivero, D. (2019). A case study on the incorporation of genderawareness into the university journalism curriculum in Spain. Gender and Education, 31(1), 1-14.

Lemus, S., Castillo, M., Moya, M., Padilla, J. L., \& Ryan, E. (2008). Elaboración y validación del Inventario de Sexismo Ambivalente para Adolescentes. International Journal of Clinical and Health Psychology, 8(2), 537-562.

León, C.M., \& Aizpurúa, E. (2020). ¿Persisten las actitudes sexistas en los estudiantes universitarios? Un análisis de su prevalencia, predictores y diferencias de género. Educación XX1, 23(1), 275-296. https://doi.org/10.5944/ educxx1.23629

Lombardo, E., \& Mergaert, L. (2013). Gender mainstreaming and resistance to gender training: A framework for studying implementation. Nordic Journal of Feministand Gender Research, 21(4), 296-311. https://doi.org/10.1080/080387 40.2013.851115

López-Vélez, A. L. (2014). La escuela inclusiva: el derecho a la equidad y la excelencia educativa. Servicio Editorial de la Universidad del País Vasco.

McCormack, O., \& Gleeson, J. (2010). Attitudes of parents of young men towards the inclusion of sexual orientation and homophobia on the Irish post-primary curriculum. Gender and Education, 22(4), 385-400.

Mateos de Cabo, R., Gimeno Nogués, R., \& Martínez Martínez, M. (junio de 2009). Presencia de estereotipos en los medios de comunicación: análisis de la prensa digital española. En Administrando en entornos inciertos. [Ponencia de Congreso] XXIII Congreso Anual AEDEM, ESIC, Sevilla.
Miralles-Cardona, C., Cardona-Moltó, M.C., \& Chiner, E. (2020). La perspectiva de género en la formación inicial docente: estudio descriptivo de las percepciones del alumnado. Educación XX1, 23(2), 231-257.

Moreno, E. (2000). La transmisión de modelos sexistas en la escuela. En M.A. Santos (Ed.), El harén pedagógico: Perspectiva de género en la organización escolar (pp.11-32). Editorial Graó.

Moya, M. (2004). Actitudes sexistas y nuevas formas de sexismo. En E. Barberá \& I. Martínez Benlloch (Eds.), Psicología y Género (pp. 271-294). Pearson.

Moya, M., \& Expósito, F. (2001). Nuevas formas, viejos intereses. Neosexismo en varones españoles. Psicothema, 13(4), 643-649

Pérez, S. (2014). Creencias sexistas y estereotipos de género en adolescentes como indicadores tempranos de violencia de género. Trabajo de campo con jóvenes residentes en Asturias [tesis de maestría]. Universidad de Oviedo, Oviedo, España.

Rebollo, M. A., Vega, L., \& García-Pérez, R. (2011). El profesorado en la aplicación de planes de igualdad: conflictos y discursos en el cambio educativo. RIE. Revista de Investigación Educativa, 29(2), 311-323.

Reinert, M. (1996). Alceste (Version 3.0). Images.

Sanchez, T. (1997) Crónica y vida de las mujeres del siglo XVI (a partir de fuentes primarias y tratados morales). Revista de Historia de la Psicología, 18(1-2), 343354.

Silva, M. J., Ferreira, E., \& Ferreira, A. (2017). Gender awareness in teacher education: Towards an embodied and inclusive approach to elementary science education. Conexão Ciência, 12(2), 147152.

Schonhardt-Bailey, C. (2013). Deliberating American monetary policy. A textual analysis. MIT Press. 
Velandia-Morales, A., \& Rincón, J. C. (2014). Estereotipos y roles de género utilizados en la publicidad transmitida a través de televisión. Universitas Psychologica, 13(2), 517-527.

Valdivieso, S., Ayuste, A., RodríguezMenéndez, M.C., \& Vila Merino, E. (2016). Educación y género en la formación docente en un enfoque de equidad y democracia. En I. Carrillo i Flores (Ed.), Democracia y educación en la formación docente (pp. 117-140). Universidad de Vic-Universidad Central de Cataluña.

Vidal, C. (2019). ¿Qué es la ideología de género, y cómo se está implantando en el mundo? [Simposio de Congreso] III
Congreso Iberoamericano por la Vida y la Familia, Panamá.

Vizcarra, M.T., Nuño, T., Lasarte, G., Aristizabal, P., \& Álvarez, A. (2015). La perspectiva de género en los títulos de grado en la Escuela Universitaria de Magisterio de Vitoria-Gasteiz. Revista de Docencia Universitaria, 13(1), 297-318. https://n9.cl/uqql

Walker, S. \& Barton, L. (1983). Gender, Class and Education. The Falmer Press.

Zippel, K., Ferree, M.M., \& Zimmermann, K. (2016). Gender equality in German Universities: Vernacularising the battle for the best brains. Gender and Education, 28(7), 867-885. 


\section{PERFIL ACADÉMICO Y PROFESIONAL DE LAS AUTORAS}

Patricia Fernández Rotaeche. ORCID: https://orcid.org/0000-0003-3682-0660

Profesora interina y doctoranda del departamento de psicología evolutiva y de la educación en la Facultad de Educación y Deporte de Álava. Línea de investigación: Sexismo y Feminismo en el ámbito educativo. E-mail: patricia.fernandezr@ehu.eus

Joana Jaureguizar Alboniga-Mayor. ORCID: https://orcid.org/0000-0001-52895967

Profesora agregada del departamento de psicología evolutiva y de la educación de la Facultad de Educación de Bilbao. IP del grupo consolidado de investigación PSIDES: Psicología del desarrollo ante los nuevos retos sociales. E-mail: joana.jauregizar@ehu.eus

Nahia Idoiaga Mondragon. ORCID: https://orcid.org/0000-0003-0345-8570

Profesora adjunta del departamento de psicología evolutiva y de la educación de la Facultad de Educación de Bilbao. Miembro del Grupo de investigación KideON, con referencia IT1342-19 (categoría A). E-mail: nahia.idoiaga@ehu.eus

Fecha Recepción del Artículo: 24. Septiembre. 2020

Fecha Modificación del Artículo: 12. Enero. 2021

Fecha Aceptación del Artículo: 13. Enero. 2021

Fecha Revisión para Publicación: 25. Febrero. 2021 